\title{
C6 Peptide Test: A Key to Diagnosis of Early Lyme disease?
}

Ritu Ramdeo Vyas*, Seon Song and Deborah S Asnis

Department of Internal Medicine, Flushing Hospital Medical Center, Flushing, NY, USA

"Corresponding author: Ritu Ramdeo Vyas, MBBS, Flushing Hospital Medical Center, Flushing, New York United States, Tel: 3012197411; E-mail: ritu.ramdeo@gmail.com

Rec date: May 27, 2014; Acc date: Jun 28, 2014; Pub date: Jul 07, 2014

Copyright: (c) 2014 Vyas RR, et al. This is an open-access article distributed under the terms of the Creative Commons Attribution License, which permits unrestricted use, distribution, and reproduction in any medium, provided the original author and source are credited.

\begin{abstract}
Lyme disease is a multisystem disease caused by infection with Borrelia. Cardiac involvement occurs during the disseminated phase of the disease, usually within weeks to a few months after the onset of infection. The most common clinical feature of Lyme carditis is atrioventricular (AV) conduction block, however, it can also cause cardiomyopathy and myopericarditis. With widespread travel, we need tests that have high sensitivity, irrespective of the borrelial species and the area where it was acquired. We believe that C6 peptide test will help us diagnose imported Lyme disease earlier in the course than the current CDC recommended two-tiered approach which might result in delayed or missed diagnosis.
\end{abstract}

Keywords: Lyme carditis; C6 peptide; European Lyme; VIsE C6 peptide

\section{Case Report}

A 69 year old woman presented with dizziness and chest pain with heart rate in thirties. She was admitted for symptomatic bradycardia. She had chronic hypertension that was treated with atenolol for years. A month before presentation, she had returned from a trip to Croatia. While abroad, she went hiking for fall foliage and felt a bite on her leg. She didn't see any tick but recalled a bulls-eye rash on the upper thigh extending to the knee followed by migratory arthralgias. She did not seek any medical attention till she developed cardiac symptoms. EKG showed 2:1 Mobitz type II AV block. Rheumatoid factor and ANA tests were negative. Progressive Lyme serology by ELISA was performed which was strongly positive at 4.23 but the western blot IgG/IgM for Borrelia burgdorferi sensu stricto was negative according to the CDC criteria. 4 out of 5 IgG bands $(23 \mathrm{kD}, 41 \mathrm{kD}, 58 \mathrm{kD}, 66 \mathrm{kD})$ and 1 out of $3 \operatorname{IgM}(23 \mathrm{kD})$ bands were positive.

There was a strong clinical suspicion for Lyme Borreliosis (LB) despite a negative western blot. Since C6 peptide testing was not available at both the hospital and the reference lab, sample was sent to the New York State Department of Health. The result came back positive along with positive western blot. The repeat western blot at $\mathrm{NYSDOH}$ was roughly six weeks after the rash. In addition to the above mentioned bands, $45 \mathrm{kD}$ IgG, $93 \mathrm{kD}$ IgG and $39 \mathrm{kD}$ IgM bands were positive, confirming the diagnosis of Lyme disease [1]. The patient received ceftriaxone initially followed by doxycycline for twenty-one days. Her AV block completely resolved [2].

\section{Discussion}

With widespread international travel, imported LB is a growing concern. While Borrelia burgdorferi is the most common species in the US, B. garinii and B. afzelii are common in Europe. Therefore, early European LB can be missed using the conventional two-tiered US assay testing. The C6 peptide corresponds to the sixth invariable area within the VIsE region, which is a conserved sequence found in Borrelia burgdorferi and the European genospecies. In early LB, ELISA followed by immunoblotting using US assays have a sensitivity of only $52 \%$ for Lyme disease acquired in Europe. Using European antigens, the sensitivity for the same increases to $81 \%$. In comparison, the sensitivity of VIsE or its C6 peptide test as a stand-alone test for diagnosing Lyme disease is $88 \%$, while US ELISA followed by a C6 ELISA as a two-tiered approach is $84 \%$ sensitive. Hence, C6 test, alone or in combination with ELISA, is superior due to higher sensitivity and similar specificity.

We hope that the newer generation assays become more readily available to include the VIsE C6 peptide. This will identify early European LB in returning travelers that can be missed due to the less sensitive US assays. In this particular case, the dilemma was to assume that the bradycardia was beta-blocker induced or due to disseminated Lyme disease as a cause the AV block. LB was later confirmed with positive western blot and treatment resulted in complete resolution of symptoms. While both C6 peptide and western blot have 100\% sensitivity in late stages, diagnosis of early LB remains a challenge.

\section{References}

1. Naila Makhani, Shaun K. Morris, Andrea V. Page, Jason Brophy, L. Robbin Lindsay, et al. (2011) A twist on Lyme: the challenge of diagnosing European Lyme neuroborreliosis. J. Clin. Microbiol 49: 455-457.

2. John A, Branda, Katy Linskey, Yeowon A. Kim, Allen C. Steere, et al. (2011) Two-Tiered Antibody Testing for Lyme Disease With Use of 2 Enzyme Immunoassays, a Whole-Cell Sonicate Enzyme Immunoassay Followed by a VlsE C6 Peptide Enzyme Immunoassay. Clinical Infectious Diseases 53: 541-547. 